\title{
Identification and Evaluation of Morpho-Physiological Variation in Spine Gourd (Momordica dioica Roxb.)
}

\author{
Sanjay Bhagat, S.K. Sinha and J.K. Tiwari* \\ Department of Genetics and Plant Breeding, RMD College of Agriculture and \\ Research Station, IGKV, Ambikapur (C.G.) 497001, India \\ *Corresponding author
}

\section{A B S T R A C T}

\begin{tabular}{|l|l|l|}
\cline { 2 - 3 } Keywords & $\begin{array}{l}\text { The present investigation was conducted with thirty four spine gourd (Momordica dioica } \\
\text { Roxb.) genotypes for evaluate their performance for various quantitative as well as } \\
\text { qualitative characters. All the qualitative characters viz. fruit colour, fruit shape, leaf } \\
\text { Cluster analysis, } \\
\text { Morphological } \\
\text { variation, Spine gourd. }\end{array}$ & $\begin{array}{l}\text { colour, leaf margin, leaf pubescence, stem colour, spine strength, conical spine density, } \\
\text { pedicel attachment with the fruit, node colour, collection site showed wide variation. } \\
\text { Among the genotypes studied. Wide range of variability was also noted with respect to } \\
\text { days to first flowering. However, with regard to quantitative character like number of fruits } \\
\text { per plant, the highest number (150) was obtained from the genotype PK-49 which was }\end{array}$ \\
\hline Article Info & $\begin{array}{l}\text { statistically similar to KRISNAPUR (138) while the lowest (54) was from PHULE MD 5- } \\
\text { 1. The genotype KRISNAPUR gave the highest yield (5982 kg/ha) and followed by the } \\
\text { yield (5868 kg/ha). Performed well in respect of studied characters under the agro climatic } \\
\text { conditions of north hill resign of Chhattisgarh. } \\
\begin{array}{ll}\text { Available Online: } \\
10 \text { November 2017 }\end{array}\end{array}$ \\
\hline \hline
\end{tabular}

\section{Introduction}

Spine gourd (Momordica dioica Roxb.) belongs to the cucurbitaceous family with chromosome number 2n=28 (Raj et al., 1993). It has originated from Indo-Malayan region (Rashid and Roy, 1976). It is distributed in Bangladesh, China, India, Malaysia, Nepal, Myanmar, Pakistan, and Sri Lanka (Rakh and Chaudhari, 2010). It is widely distributed in tropical and sub-tropical parts of India and adapted to different soil and climatic conditions (Basumatary et al., 2014). In Chhattisgarh distributed in baster plateau zone and northern hill region in forest areas and largely cultivated in tribal regions farmer but no study in area and production and productivity. It is economically important vegetable plant with high food and medicinal value, cultivated for its fruits, which is used as vegetable and is known by various names such as Kakrol, Kartoli, and Kankoda, Teasel gourd or Bhat Kerala. Per $100 \mathrm{~g}$ edible fruit was found to contain $84.1 \%$ moisture, $7.7 \mathrm{~g}$ carbohydrate, $3.1 \mathrm{~g}$ protein, $3.1 \mathrm{~g}$ fat, $3.0 \mathrm{~g}$ fiber and $1.1 \mathrm{~g}$ minerals. It also contained small quantities of essential vitamins like ascorbic acid, carotene, thiamin, riboflavin and niacin (Kushwaha et al., 2005) This popular vegetable has high demand in market because of good nutritional, medicinal value, high keeping quality ability to withstand long distance transportation, high market price and good export potential (Rasul, 2003). In spite 
of many advantages, there was no research thrust paid on Spine guard improvement, genetic diversity or genetic relatedness and its utilization in breeding programme based on morphological and physiological variation have been studied in many crops (Masud et al., 1995; Chowdhury et al., 1998; Rahman et al., 2001; Sultana et al., 2001; Grent et al., 2001; Brown and Myers, 2002). The knowledge on nature and magnitude of genetic variability existed in Spine guard will provide the foundation of designing breeding programme. So, persent investigation was carried out to evaluate morpho-physiological variation in spine guard.

\section{Materials and Methods}

Tuberous roots of 34 genotypes namely included checked varieties Indira Kankoda-1, PK -5, PK -9, PK -26, PK-34, PK-35, PK 46, KRISHNAPUR, PK-49, RMF-1, RMF17, RMF-27, RMF-P-4, RMF-7-P-1, PHULLE MD-5-1, PHULLE MD-5-2, NDM1, NDM-5, RMDSG-1, PK -33, AMBIKA-K12-1, AMBIKA 13-5, AMBIKA 13-6, RAIGARH， NDM-2, NDM-3， NDM-4, DHARMJAYGARH， RMF-G-39， RMF-G49, AJSG-3, AJSG-4, AJSG-5, of Momordica dioica were collected from different agroecological regions of Chhattisgarh in during 1998 to July 2016. They were maintained at the experimental farm of Rajmohini Devi College of Agriculture and Research Station Ambikapur Chhattisgarh.

The experiment was conducted consequently for two year during 2015 and 2016 under All India Coordinated Research Network Project on Potential crops at the research and instructional farm of Rajmohini Devi College of Agriculture and Research Station, Ambikapur. Experiment was conducted in Augmented Block design with four blocks; pits of $30 \times 30 \times 30 \mathrm{~cm}^{3}$ were prepared in each plot with a spacing of $2 \mathrm{~m} \times 2 \mathrm{~m}$. The tubers root was planted in earthen pots containing a 2:1:1 mixture of soil sand and decomposed cow dung on May 2015 and watered. They began to sprouted 20-25 days after potting. The male plants were planted in the field at 8:1 ratio (female: male). The plants were supported by bamboo sticks. When the plants were about $2.5 \mathrm{~m}$. high, they were allowed to climb on rope net hanged vertically up to $2.5 \mathrm{~m}$ from the soil surface.

Twenty eight parameters of morphological and physiological characteristics as listed in table 1 were reported from all plants. A data matrix was generated from the parameters of characteristics listed in table 1. Similarity matrix was generated using the SimQual programme NTSYSpc software version 2.02 (Rohlf, 1998). The similarity coefficients were used for cluster analysis and dendogram was constructed by Unweighted Pair-Group Method with Arithmetic Average (UPGMA) (Mathew et al., 2000) (Fig. 1).

\section{Results and Discussion}

Twenty eight morphological traits recorded among spine guard genotypes are furnished in table 2. The stem colour for the spine guard genotypes from light green to green in colour. Genotypes INDIRA KANKODA-1, PK -5, PK -9, PK -26, PK-34, PK -46, KRISHNAPUR, PK-49, RMF1, RMF17, RMF-27, RMF-P-4, RMF-7-P-1, PHULLE MD-5-1, PHULLE MD-5-2, NDM-5, RMDSG-1, PK -33, AMBIKA-K12-1, AMBIKA, 13-5, AMBIKA 13-6, RAIGARH, NDM-2, NDM-3, NDM-4, DHARMJAYGARH, RMF-G-39, RMF-G49, AJSG-3, AJSG-4, and AJSG-5 had light green stem colour while genotypes PK-35 and NDM-1exhibited to green colour.

The leaf intensity of green colour in leaf ranged from green to light green in different genotypes Indira Kankoda-1, PK -5, PK -26, 
PK-34, PK -46, PK-49, RMF-1, RMF-17, RMF-27, RMF-P-4, RMF-7-P-1, PHULLE MD-5-1, PHULLE MD-5-2, NDM-5, RMDSG-1, PK -33, AMBIKA-K-12-1, AMBIKA, 13-5,AMBIKA 13-6, RAIGARH, NDM-2, NDM-3, NDM-4, DHARMJAYGARH， RMF-G-39， RMF-G49, AJSG-3, AJSG-4, and AJSG-5 had light green while, it was recorded as green genotypes in PK -9, PK-35, KRISHNAPUR, and NDM-1.

Leaf length highest in genotypes PK-46 and lowest leaves length PK-33. Leaf width highest recorded in genotypes PK-46 and lowest width PK-33. Leaves margin may be characterized serrated leaves (5 Lobes) PK-9, NDM-1, NDM-2, NDM-3, NDM-4, NDM-5, and RMF-G-49, dented leaves (4 Lobes) PK34, PK- 49, DHARAMJAYGARH, RMF-G39, and AJSG-5, slightly dented leaves coding (3 Lobes) PK-5, PK-26, PK-35, RMF-1 and AJSG-4, and leaf margin absent INDIRA KANKODA-1， PK -46, KRISHNAPUR, RMF-17, RMF-27, RMF-P-4, RMF-7-P-1, PHULLE MD-5-1, PHULLE MD-5-2, RMDSG-1， PK -33， AMBIKA-K-12-1, AMBIKA 13-5, AMBIKA 13-6, RAIGARH and AJSG-5.

The pubescence of leaf surface was found to be weak PK -5, PK -9, PK -26, PK -46, KRISHNAPUR, PK-49, RMF-1, RMF-17, RMF-27, PHULLE MD-5-1, PHULLE MD5-2, NDM-5, RMDSG-1, PK -33, RAIGARH, NDM-4, DHARMJAYGARH， RMF-G-39, RMF-G-49, and AJSG-4 and it was absent in INDIRA KANKODA-1, PK-34, RMF-P-4, RMF-7-P-1, AMBIKA-K12-1, AMBIKA 135, AMBIKA 13-6, NDM-2, NDM-1, NDM-3, AJSG-3 and AJSG-5.

There was no difference observed in green sepal and petal colors. Flower characteristics included pedicel length, ovary length and diameter, style length and pistil tip length, pedicel length divided into the three categories
(Short, Medium, and Long) but result found in all genotypes short.

The longer the pedicel length, the easier to pick the fruit up during harvest. Fruit with short pedicel usually are hidden under the leaves and cause problems during harvest (generally fruit are hanged under the net). All genotypes had small ovary length and diameter. Almost all the genotype showed medium style and pistil tip length while genotypes AMBIKA 13-5, RMDSG-1, NDM4, PHULE MD-5-1, NDM- 5, PK-33, PK-34, RMF-G-49 and AJSG -4 has long style.

The color of fruit during edible maturity showed great variability among the genotypes and categorized into greenish yellow, yellow green, green, dark green, and light green. The number of genotypes fallen under each two groups $1^{\text {st }}$ groups green fruit colour INDIRA KANKODA-1, PK- 5, PK-9, PK-26, PK-34, PK-46, KRISHNAPUR, RMF-1, RMF-7-P-1, PHULE MD 5-1, NDM-5, RMDSG-1, NDM3, AJSG-3 and AJSG-5 genotypes and $2^{\text {nd }}$ groups light green colour PK-35, PK-49, RMF-17, RMF-27, RMF P-4, PHULE MD-52, NDM-1, PK-33, AMBIKA K 12-1, AMBIKA 13-5, AMBIKA 13-6, RAIGARH, NDM-2, NDM-4, DHARAMJAYGARH, RMF-G-39, RMF-G-49 and AJSG-4 genotypes.

Fruit shape had also shown much variability among the genotypes such as round KRISHNAPUR, RMF-1, RMF-27, RMF-7-P1, PHULE-MD-5-2, AMBIKA-13-5, RAIGARH, RMF-G-39, AJSG-3 AJSG-4 and AJSG-5 and oval genotypes INDIRA KANKODA-1, PK-5, PK-9, PK-26, PK-35, PK-35, PK-46, PK-49, RMF-17, RMF P-4, PHULE MD 5-1, NDM-1, NDM-5, RMDSG1, PK-33, AMBIKA-K 12-1, AMBIKA-13-6, NDM-2, NDM-3, NDM-4, DHARAMJAYGARH, and RMF-G-49 (Fig. 2). 
Fig.1 Dendrogram of 34 genotypes constructed by morphological characterization using UPGMA based on Euclidean dissimilarity values

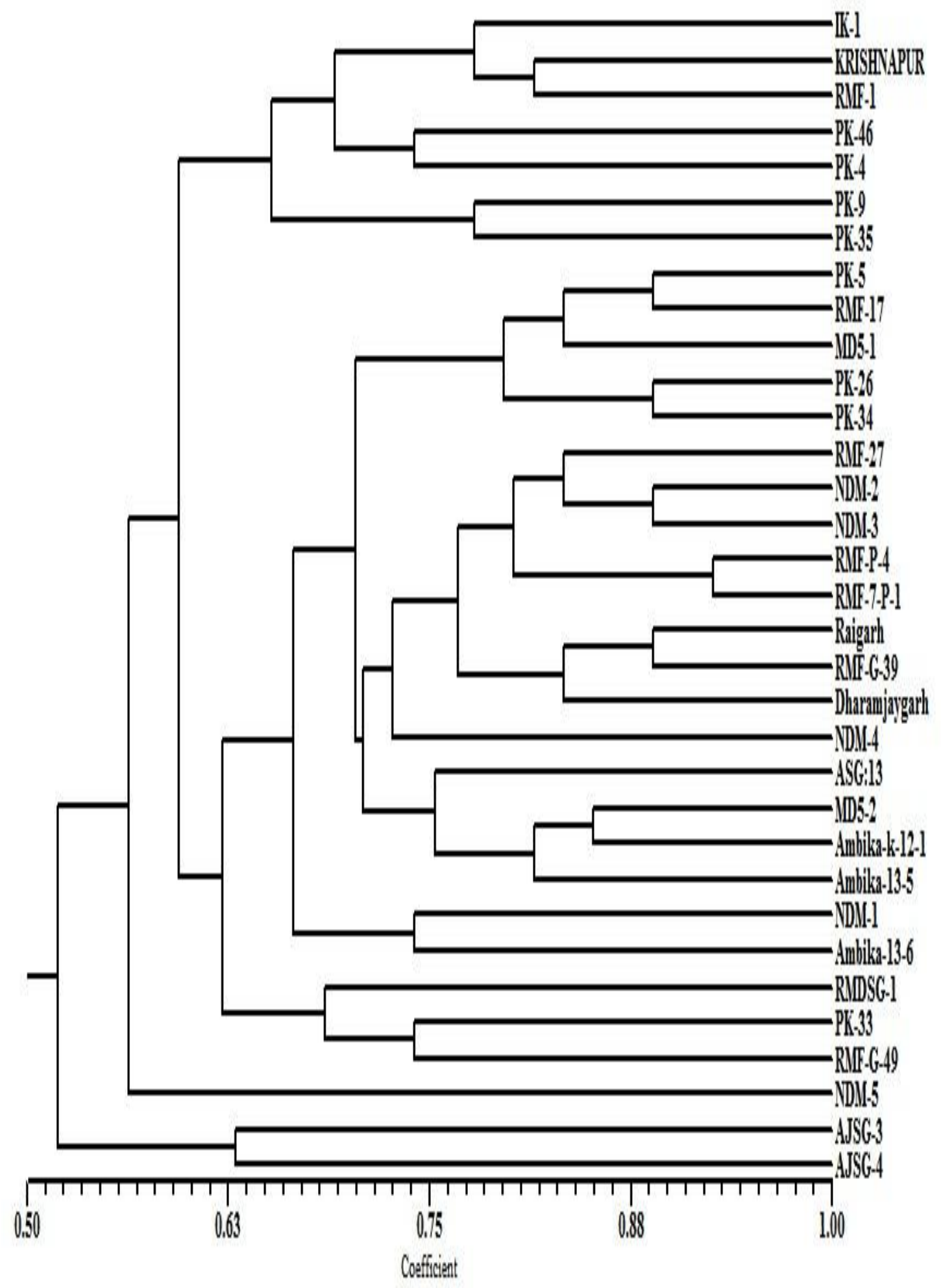


Fig.2 Fruit spine guard

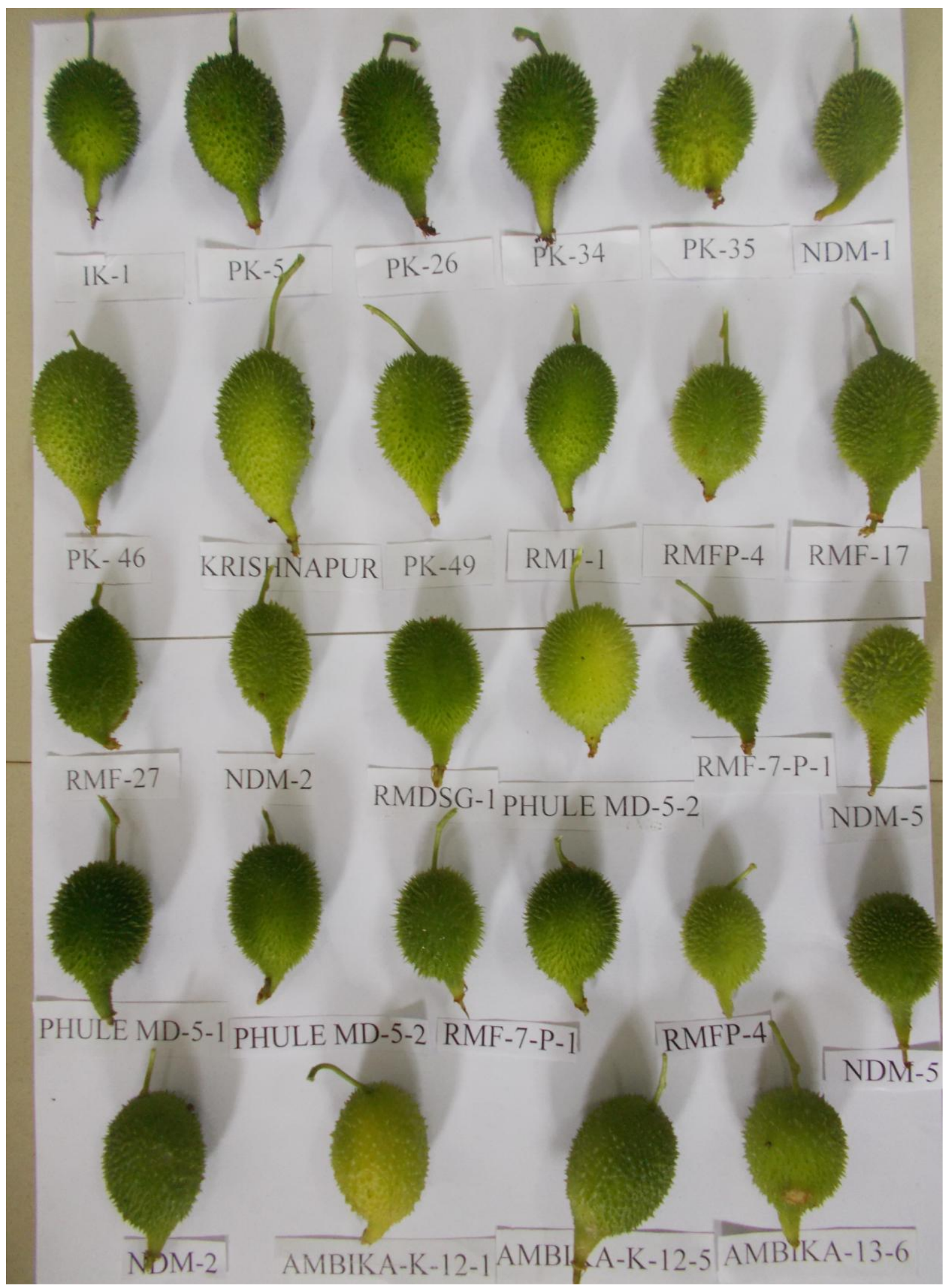


Table.1 Parameters (descriptor) of morphological and physiological characters studies in spine gourd

\begin{tabular}{|c|c|c|c|c|c|}
\hline S.N. & ITEM & PARAMETER & S.N. & ITEM & PARAMETER \\
\hline \multirow[t]{2}{*}{1} & \multirow{2}{*}{$\begin{array}{l}\text { Days of first } \\
\text { flowering }\end{array}$} & \multirow{2}{*}{$\begin{array}{l}\text { 1.Early ( } 60-80 \text { days) } \\
\text { 3.Medium early ( } 81-100 \text { days) } \\
\text { 5. Medium ( } 101-120 \text { days) } \\
\text { 7.Late ( } 121-140 \text { days })\end{array}$} & 15 & Style length & $\begin{array}{l}\text { 1. Short }(<6 \mathrm{~mm}) \\
\text { 5. Medium }(6.1-9 \mathrm{~mm}) \\
\text { 9. Long }(>9.1 \mathrm{~mm}) \\
\end{array}$ \\
\hline & & & \multirow[b]{2}{*}{16} & \multirow[b]{2}{*}{ Pistil tip length } & \multirow[b]{2}{*}{$\begin{array}{l}\text { 1. Short }(<4 \mathrm{~mm}) \\
\text { 5. Medium }(4.1-6.0 \mathrm{~mm}) \\
\text { 9. Long }(>6.1 \mathrm{~mm})\end{array}$} \\
\hline \multirow[t]{2}{*}{2} & \multirow[b]{2}{*}{$\begin{array}{l}\text { Number of first } \\
\text { flowering node }\end{array}$} & \multirow{2}{*}{$\begin{array}{l}\text { 1. } 1-10 \text { nodes } \\
\text { 2. } 11-20 \text { nodes } \\
\text { 3. } 21-30 \text { nodes } \\
\text { 4. } 31-40 \text { nodes } \\
\text { 5. } 41-50 \text { nodes } \\
\text { 6.51-60 nodes } \\
\text { 7. } 61-70 \text { nodes }\end{array}$} & & & \\
\hline & & & \multirow[t]{2}{*}{17} & \multirow[t]{2}{*}{ Fruit colour } & $\begin{array}{l}\text { 1. Greenish yellow }(\mathrm{GY}) \\
\text { 3. Yellow green }(\mathrm{YG}) \mathrm{s} \\
\text { 5. Green }(\mathrm{G}) \\
\text { 7. Dark green }(\mathrm{DG})\end{array}$ \\
\hline \multirow[t]{2}{*}{3} & \multirow{2}{*}{ Stem colour } & \multirow{2}{*}{$\begin{array}{l}\text { 1. Light green (L.G) } \\
\text { 3. Green (G.) } \\
\text { 5. Dark green(D.G.) } \\
\end{array}$} & & & $\begin{array}{l}\text { 9. Light green orange(LG) } \\
\text { 11.Light orange }(\mathrm{LO})\end{array}$ \\
\hline & & & \multirow{3}{*}{18} & \multirow{3}{*}{ Fruit shape } & 1. Round (R) \\
\hline 4 & $\begin{array}{l}\text { No. of ridge on } \\
\text { stem }\end{array}$ & $\begin{array}{l}\text { 1. Persent ( } 5 \text { ridge) } \\
\text { 9. Absence no ridge }\end{array}$ & & & $\begin{array}{l}\text { 3. Oval }(\mathrm{OV}) \\
\text { 5. Cylindrical }(\mathrm{C})\end{array}$ \\
\hline \multirow{3}{*}{5} & \multirow{3}{*}{$\begin{array}{l}\text { Node colour at the } \\
\text { attachment of the } \\
\text { leaves }\end{array}$} & \multirow{3}{*}{$\begin{array}{l}\text { 1. Light green (L.G) } \\
\text { 3. Green (G.) } \\
\text { 5. Dark green(D.G.) } \\
\text { 7. Blackish green(B.G) }\end{array}$} & & & $\begin{array}{l}\text { 7. Oblong }(\mathrm{O}) \\
\text { 9. Spindle (S) }\end{array}$ \\
\hline & & & 19 & Conical spine density & $\begin{array}{l}\text { 1. Thin (T) } \\
\text { 3. Thick (TK) }\end{array}$ \\
\hline & & & \multirow[t]{2}{*}{20} & \multirow{2}{*}{$\begin{array}{l}\text { Conical spine } \\
\text { strength }\end{array}$} & 1. Soft (S) \\
\hline \multirow[b]{2}{*}{6} & \multirow[b]{2}{*}{ No. stem per plant } & \multirow{2}{*}{$\begin{array}{l}\text { 1. Few }(<10 \text { stems }) \\
\text { 5. Moderate }(11-20 \text { stems }) \\
\text { 9. Many }(>21 \text { stems }) \\
\end{array}$} & & & 3. Hard $(\mathrm{H})$ \\
\hline & & & \multirow{2}{*}{21} & \multirow{2}{*}{$\begin{array}{l}\text { Pedicel attachment } \\
\text { with the fruit }\end{array}$} & $\begin{array}{l}\text { 1. Depressed (D) } \\
\text { 3. Slightly depressed(SD) }\end{array}$ \\
\hline \multirow[b]{2}{*}{7} & \multirow[b]{2}{*}{ Leaf color } & \multirow{2}{*}{$\begin{array}{l}\text { 1. Light green (L.G) } \\
\text { 3. Green (G.) } \\
\text { 5. Dark green(D.G.) }\end{array}$} & & & 5. Pointed $(\mathrm{P})$ \\
\hline & & & \multirow[t]{2}{*}{22} & \multirow[t]{2}{*}{ fruit length } & $\begin{array}{l}\text { 1. Short }(<50 \mathrm{~mm}) \\
\text { 3. Medium }(51-90 \mathrm{~mm})\end{array}$ \\
\hline \multirow[t]{2}{*}{8} & \multirow[t]{2}{*}{ Leaf length } & $\begin{array}{l}\text { 1. Short }(1-7 \mathrm{~cm}) \\
\text { 5. Medium }(7.1-15 \mathrm{~cm})\end{array}$ & & & $\begin{array}{l}\text { 5. Long }(>91-130 \mathrm{~mm}) \\
\text { 7. Very long }(>131)\end{array}$ \\
\hline & & 9. Long $(>15.1 \mathrm{~cm})$ & & & 1. Small $(1-40 \mathrm{~mm})$ \\
\hline 9 & Leaf width & $\begin{array}{l}\text { 1. Narrow }(1-7 \mathrm{~cm}) \\
\text { 5. Medium }(7.1-15 \mathrm{~cm})\end{array}$ & 23 & Fruit diameter & $\begin{array}{l}\text { 5. Medium(41-80mm) } \\
\text { 9. Large }(81-120 \mathrm{~mm})\end{array}$ \\
\hline & & 9. Wide $(>15.1 \mathrm{~cm})$ & & & 1. Light $(1-50 \mathrm{~g})$ \\
\hline & Leaf margin & $\begin{array}{l}\text { 1. Absence(no attachment) } \\
\text { 3. Slightly dented (SD) } \\
\text { 5. Medium dented (MD) }\end{array}$ & 24 & Single fruit weight & $\begin{array}{l}\text { 3. Medium }(51-100 \mathrm{~g}) \\
\text { 5. Heavy }(101-150 \mathrm{~g}) \\
\text { 7. Very heavy }(>151 \mathrm{~g}) \\
\end{array}$ \\
\hline 10 & & $\begin{array}{l}\text { 7. Dented(D) } \\
\text { 9. Serrated (5 lobes) }\end{array}$ & & & $\begin{array}{l}\text { 1. Few }(<20) \\
\text { 3. Moderate }(21-41)\end{array}$ \\
\hline 11 & Leaf pubescence & $\begin{array}{l}\text { 1. Absence(Ab) } \\
\text { 3. Few (F) }\end{array}$ & 25 & $\begin{array}{l}\text { No. of fruits per } \\
\text { plant }\end{array}$ & $\begin{array}{l}\text { 5. Many }(41-60) \\
\text { 7. Profuse }(>61)\end{array}$ \\
\hline & & $\begin{array}{l}\text { 5. Medium(M) } \\
\text { 7. More }(\mathrm{Mo}) \\
\end{array}$ & & & $\begin{array}{l}\text { 1. Low }(<1.0 \mathrm{~kg}) \\
\text { 3. Medium }(1.1-2.0 \mathrm{~kg})\end{array}$ \\
\hline 12 & Pedicel length & $\begin{array}{l}\text { 1. Short }(1-7 \mathrm{~cm}) \\
\text { 5. Medium }(7.1-15 \mathrm{~cm}) \\
\text { 9. Long }(>15.1 \mathrm{~cm})\end{array}$ & 26 & Fruit yield per plant & $\begin{array}{l}\text { 5. Good }(2.1-3.0 \mathrm{~kg}) \\
\text { 7. Very good }(3.1-4.0 \mathrm{~kg}) \\
\text { 9. Bumper }(>4.1 \mathrm{~kg})\end{array}$ \\
\hline 13 & Ovary length & $\begin{array}{l}\text { 1. Short }(>1-10 \mathrm{~mm}) \\
\text { 5. Medium }(11-20 \mathrm{~mm}) \\
\text { 9. Long }(>21 \mathrm{~mm}) \\
\end{array}$ & 27 & No seed per fruit & $\begin{array}{l}\text { 1. Few (1-15) } \\
\text { 3. Less(16-30) } \\
\text { 5. Medium (31-45) }\end{array}$ \\
\hline & & 1. Small $(<7 \mathrm{~mm})$ & & & 7. Many (46-60) \\
\hline 14 & Ovary diameter & 5. Large $(>7.1 \mathrm{~mm})$ & 28 & 100 Seed weight & $\begin{array}{l}\text { 1. Light }(<10 \mathrm{~g}) \\
\text { 3. Slightly bold }(21 \mathrm{~g}-40 \mathrm{~g}) \\
\text { 5. Bold }(>40 \mathrm{~g})\end{array}$ \\
\hline
\end{tabular}


Table.2 Morphological characterization of spine guard genotypes based on descriptor

\begin{tabular}{|c|c|c|c|c|c|c|c|c|c|c|c|c|c|c|c|c|c|c|c|c|c|c|c|c|c|c|c|c|c|c|c|c|}
\hline Parameters & 1 & 2 & \begin{tabular}{|l|l} 
& \\
\end{tabular} & 4 & \begin{tabular}{c|c}
5 & 6 \\
\end{tabular} & \begin{tabular}{l|l|}
6 & 7 \\
\end{tabular} & \begin{tabular}{|l|l|}
8 & 9 \\
\end{tabular} & $\begin{array}{l}9 \\
9\end{array}$ & 11 & 12 & 13 & 14 & 15 & 16 & 17 & 18 & 19 & 20 & 21 & 22 & 23 & 24 & 25 & 26 & 27 & 28 & 29 & 30 & 31 & 32 & 33 & 34 \\
\hline Days to first flowering & 1 & 1 & \begin{tabular}{|l|l}
1 & \\
\end{tabular} & 1 & \begin{tabular}{l|l}
1 & 1
\end{tabular} & \begin{tabular}{|l|l|}
1 & 1 \\
\end{tabular} & \begin{tabular}{|l|l|l}
1 & 1
\end{tabular} & \begin{tabular}{l|l|}
1 & 1 \\
\end{tabular} & 1 & 1 & 1 & 1 & 1 & 1 & 1 & 1 & 1 & 1 & 1 & 1 & 1 & 1 & 1 & 1 & 1 & 1 & 1 & 1 & 1 & 1 & 1 & 1 \\
\hline No of first flowering node & 1 & 2 & \begin{tabular}{|c|c|c|}
2 & \\
\end{tabular} & 1 & \begin{tabular}{l|l}
2 & 2 \\
\end{tabular} & \begin{tabular}{l|l|}
2 & 1 \\
\end{tabular} & \begin{tabular}{|l|l|}
3 & 2 \\
\end{tabular} & 2 & 3 & 2 & 1 & 1 & 1 & 1 & 2 & 2 & 3 & 2 & 2 & 2 & 3 & 2 & 2 & 2 & 2 & 3 & 2 & 2 & 1 & 1 & 2 & 3 \\
\hline Stem colour & 1 & 1 & \begin{tabular}{|l|l}
1 &
\end{tabular} & 1 & \begin{tabular}{l|l}
1 & 3
\end{tabular} & \begin{tabular}{|l|l|}
3 & 1 \\
\end{tabular} & \begin{tabular}{|l|l|l}
1 & 1 \\
\end{tabular} & \begin{tabular}{l|l|}
1 & 1 \\
\end{tabular} & 1 & 1 & 1 & 1 & 1 & 1 & 3 & 1 & 1 & 1 & 1 & 1 & 1 & 1 & 1 & 1 & 1 & 1 & 1 & 1 & 1 & 1 & 1 & 1 \\
\hline No. of ridge on stem colour & 1 & 9 & \begin{tabular}{|l|l}
1 & \\
\end{tabular} & 1 & \begin{tabular}{l|l}
9 & 9 \\
\end{tabular} & \begin{tabular}{l|l|}
9 & 1 \\
\end{tabular} & \begin{tabular}{|l|l|}
9 & 9 \\
\end{tabular} & \begin{tabular}{l|l}
9 & 9 \\
\end{tabular} & 1 & 1 & 1 & 1 & 1 & 9 & 1 & 1 & 1 & 9 & 9 & 1 & 1 & 9 & 1 & 9 & 9 & 1 & 9 & 9 & 1 & 1 & 1 & 9 \\
\hline Node colour at the attachment of the leaves & 1 & 1 & \begin{tabular}{|l|l}
1 & \\
\end{tabular} & 1 & \begin{tabular}{l|l}
1 & 1 \\
\end{tabular} & 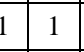 & \begin{tabular}{|l|l|}
1 & 1 \\
\end{tabular} & \begin{tabular}{l|l}
1 & 1 \\
\end{tabular} & 1 & 1 & 1 & 1 & 1 & 1 & 1 & 1 & 1 & 1 & 1 & 1 & 1 & 1 & 1 & 1 & 1 & 1 & 1 & 1 & 1 & 1 & 1 & 1 \\
\hline No. of stem per plant & 1 & 1 & \begin{tabular}{|l|l}
5 & \\
\end{tabular} & 1 & \begin{tabular}{l|l}
1 & 1 \\
\end{tabular} & 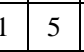 & \begin{tabular}{|l|l|}
5 & 1 \\
\end{tabular} & \begin{tabular}{l|l}
1 & 1 \\
\end{tabular} & 1 & 1 & 1 & 1 & 1 & 1 & 1 & 1 & 5 & 5 & 5 & 5 & 1 & 1 & 1 & 1 & 1 & 5 & 1 & 1 & 1 & 1 & 1 & 1 \\
\hline Leaf colour & 1 & 1 & 3 & 1 & \begin{tabular}{l|l}
1 & 3 \\
\end{tabular} & \begin{tabular}{l|l|}
3 & 1 \\
\end{tabular} & \begin{tabular}{|l|l|}
1 & 1 \\
\end{tabular} & \begin{tabular}{l|l}
1 & 1 \\
\end{tabular} & 1 & 1 & 1 & 1 & 1 & 1 & 3 & 1 & 1 & 1 & 1 & 1 & 1 & 1 & 1 & 1 & 1 & 1 & 1 & 1 & 1 & 1 & 1 & 1 \\
\hline Leaf length $(\mathrm{cm})$ & 5 & 1 & \begin{tabular}{|l|l}
5 & \\
\end{tabular} & 1 & \begin{tabular}{l|l}
1 & 5
\end{tabular} & \begin{tabular}{|l|l|}
5 & 5 \\
\end{tabular} & \begin{tabular}{|l|l|}
5 & 5 \\
\end{tabular} & \begin{tabular}{|l|l|}
5 & 5
\end{tabular} & 1 & 1 & 1 & 1 & 1 & 1 & 1 & 1 & 1 & 5 & 1 & 5 & 5 & 1 & 1 & 1 & 1 & 1 & 1 & 1 & 5 & 1 & 5 & 1 \\
\hline Leaf width $(\mathrm{cm})$ & 5 & 1 & 5 & 1 & \begin{tabular}{l|l}
5 & 5 \\
\end{tabular} & \begin{tabular}{l|l|}
5 & 5 \\
\end{tabular} & \begin{tabular}{|l|l|}
5 & 1 \\
\end{tabular} & $1 \quad 5$ & 1 & 1 & 1 & 1 & 1 & 1 & 1 & 1 & 1 & 1 & 5 & 1 & 5 & 1 & 1 & 1 & 1 & 1 & 1 & 1 & 1 & 1 & 1 & 1 \\
\hline Leaf margin & 1 & 3 & \begin{tabular}{|l|l}
9 & 1 \\
\end{tabular} & 3 & \begin{tabular}{l|l}
7 & 3 \\
\end{tabular} & \begin{tabular}{|l|l|}
3 & 1 \\
\end{tabular} & \begin{tabular}{|l|l|}
1 & 7 \\
\end{tabular} & \begin{tabular}{l|l|}
7 & 3 \\
\end{tabular} & 1 & 1 & 1 & 1 & 1 & 1 & 1 & 9 & 9 & 1 & 1 & 1 & 1 & 1 & 1 & 9 & 9 & 7 & 7 & 7 & 9 & 7 & 3 & 1 \\
\hline Leaf pubescence & 3 & 3 & \begin{tabular}{|l|l}
3 & \\
\end{tabular} & 3 & \begin{tabular}{l|l}
1 & 3 \\
\end{tabular} & \begin{tabular}{|l|l|}
3 & 3 \\
\end{tabular} & \begin{tabular}{|l|l|}
1 & 3 \\
\end{tabular} & \begin{tabular}{|l|l|}
3 & 3 \\
\end{tabular} & 3 & 3 & 3 & 1 & 1 & 3 & 3 & 1 & 3 & 3 & 3 & 1 & 1 & 1 & 3 & 1 & 1 & 3 & 3 & 3 & 3 & 1 & 3 & 1 \\
\hline Pedicel length $(\mathrm{cm})$ & 1 & 1 & \begin{tabular}{|l|l}
1 &
\end{tabular} & 1 & \begin{tabular}{l|l}
1 & 1
\end{tabular} & \begin{tabular}{|l|l|}
1 & 1 \\
\end{tabular} & \begin{tabular}{|l|l|}
1 & 1 \\
\end{tabular} & \begin{tabular}{l|l|}
1 & 1 \\
\end{tabular} & 1 & 1 & 1 & 1 & 1 & 1 & 1 & 1 & 1 & 1 & 1 & 1 & 1 & 1 & 1 & 1 & 1 & 1 & 1 & 1 & 1 & 1 & 1 & 1 \\
\hline Ovary length $(\mathrm{cm})$ & 1 & 1 & \begin{tabular}{|l|l}
1 & \\
\end{tabular} & 1 & \begin{tabular}{l|l}
1 & 1 \\
\end{tabular} & 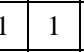 & \begin{tabular}{|l|l|}
1 & 1 \\
\end{tabular} & \begin{tabular}{l|l|}
1 & 1 \\
\end{tabular} & 1 & 1 & 1 & 1 & 1 & 1 & 1 & 1 & 1 & 1 & 1 & 1 & 1 & 1 & 1 & 1 & 1 & 1 & 1 & 1 & 1 & 1 & 1 & 1 \\
\hline Ovary diameter $(\mathrm{cm})$ & 1 & 1 & \begin{tabular}{|l|l}
1 & \\
\end{tabular} & 1 & \begin{tabular}{l|l}
1 & 1 \\
\end{tabular} & 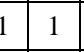 & \begin{tabular}{|l|l|}
1 & 1 \\
\end{tabular} & \begin{tabular}{l|l}
1 & 1 \\
\end{tabular} & 1 & 1 & 1 & 1 & 1 & 1 & 1 & 1 & 1 & 1 & 1 & 1 & 1 & 1 & 1 & 1 & 1 & 1 & 1 & 1 & 1 & 1 & 1 & 1 \\
\hline Style length $(\mathrm{cm})$ & 5 & 5 & \begin{tabular}{|l|l}
5 & 2
\end{tabular} & 5 & \begin{tabular}{l|l}
5 & 5
\end{tabular} & \begin{tabular}{|l|l|}
5 & 5
\end{tabular} & \begin{tabular}{|l|l|}
5 & 5 \\
\end{tabular} & \begin{tabular}{|l|l|}
5 & 5
\end{tabular} & 5 & 5 & 5 & 5 & 5 & 5 & 9 & 5 & 9 & 9 & 9 & 5 & 9 & 5 & 5 & 5 & 5 & 9 & 1 & 9 & 9 & 5 & 9 & 5 \\
\hline Pistil length $(\mathrm{cm})$ & 1 & 5 & \begin{tabular}{l|l}
1 & \\
\end{tabular} & 5 & \begin{tabular}{l|l}
5 & 1 \\
\end{tabular} & \begin{tabular}{|l|l|}
1 & 5 \\
\end{tabular} & \begin{tabular}{|l|l|}
5 & 5 \\
\end{tabular} & \begin{tabular}{l|l|}
5 & 1 \\
\end{tabular} & 9 & 9 & 1 & 5 & 9 & 1 & 5 & 1 & 9 & 5 & 1 & 1 & 1 & 1 & 5 & 5 & 5 & 5 & 5 & 5 & 9 & 5 & 5 & 5 \\
\hline Fruit colour & 5 & 5 & \begin{tabular}{|l|l}
5 & \\
\end{tabular} & 5 & \begin{tabular}{l|l}
5 & 9 \\
\end{tabular} & \begin{tabular}{l|l}
9 & 5 \\
\end{tabular} & \begin{tabular}{|l|l|}
5 & 9 \\
\end{tabular} & \begin{tabular}{|l|l|}
9 & 5 \\
\end{tabular} & 9 & 9 & 5 & 9 & 5 & 5 & 9 & 9 & 5 & 5 & 9 & 9 & 9 & 9 & 9 & 9 & 5 & 9 & 9 & 9 & 9 & 5 & 9 & 5 \\
\hline Fruit shape & 3 & 3 & 3 & 3 & \begin{tabular}{l|l}
3 & 3 \\
\end{tabular} & \begin{tabular}{l|l}
3 & 3 \\
\end{tabular} & \begin{tabular}{|l|l|}
1 & 3 \\
\end{tabular} & 1 & 3 & 1 & 1 & 3 & 1 & 3 & 3 & 3 & 3 & 3 & 3 & 3 & 1 & 3 & 1 & 3 & 3 & 3 & 3 & 1 & 3 & 1 & 1 & 1 \\
\hline Conical spine density & 3 & 3 & \begin{tabular}{|l|l}
3 &
\end{tabular} & 3 & \begin{tabular}{l|l}
1 & 3
\end{tabular} & \begin{tabular}{l|l}
33 & 1 \\
\end{tabular} & \begin{tabular}{|l|l|}
3 & 1 \\
\end{tabular} & \begin{tabular}{l|l|}
1 & 3 \\
\end{tabular} & 1 & 1 & 3 & 1 & 3 & 3 & 3 & 3 & 3 & 1 & 1 & 3 & 3 & 3 & 3 & 3 & 1 & 3 & 3 & 3 & 3 & 1 & 1 & 1 \\
\hline Conical spine strength & 3 & 3 & \begin{tabular}{|l|l}
3 & 1 \\
\end{tabular} & 3 & \begin{tabular}{l|l}
1 & 3
\end{tabular} & \begin{tabular}{l|l|l|}
3 & 1 &
\end{tabular} & \begin{tabular}{|l|l|}
3 & 1 \\
\end{tabular} & $1 \quad 3$ & 1 & 1 & 3 & 1 & 3 & 3 & 3 & 3 & 3 & 1 & 1 & 3 & 3 & 3 & 3 & 3 & 1 & 3 & 3 & 3 & 3 & 1 & 1 & 1 \\
\hline Pedicel attachment with the fruit & 5 & 5 & \begin{tabular}{l|l}
5 &
\end{tabular} & 5 & \begin{tabular}{l|l}
5 & 5
\end{tabular} & \begin{tabular}{l|l}
5 & 5
\end{tabular} & \begin{tabular}{|l|l|}
5 & 5 \\
\end{tabular} & \begin{tabular}{|l|l|}
5 & 5 \\
\end{tabular} & 5 & 5 & 5 & 5 & 5 & 5 & 5 & 5 & 5 & 5 & 5 & 5 & 5 & 5 & 5 & 5 & 5 & 5 & 5 & 5 & 5 & 5 & 5 & 5 \\
\hline Fruit length $(\mathrm{cm})$ & 1 & 1 & \begin{tabular}{|l|l}
1 & \\
\end{tabular} & 1 & \begin{tabular}{l|l}
3 & 1 \\
\end{tabular} & \begin{tabular}{l|l}
1 & 1 \\
\end{tabular} & \begin{tabular}{|l|l|}
1 & 3 \\
\end{tabular} & \begin{tabular}{|l|l|}
3 & 1 \\
\end{tabular} & 1 & 1 & 1 & 1 & 1 & 1 & 1 & 1 & 3 & 1 & 1 & 1 & 1 & 1 & 1 & 1 & 1 & 1 & 1 & 1 & 1 & 1 & 1 & 1 \\
\hline Fruit diameter $(\mathrm{cm})$ & 1 & 1 & \begin{tabular}{|l|l}
1 & \\
\end{tabular} & 1 & \begin{tabular}{l|l}
1 & 1 \\
\end{tabular} & \begin{tabular}{|l|l|}
1 & 1 \\
\end{tabular} & \begin{tabular}{|l|l|}
1 & 1 \\
\end{tabular} & \begin{tabular}{l|l|}
1 & 1 \\
\end{tabular} & 1 & 1 & 1 & 1 & 1 & 1 & 1 & 1 & 1 & 1 & 1 & 1 & 1 & 1 & 1 & 1 & 1 & 1 & 1 & 1 & 1 & 1 & 1 & 1 \\
\hline Single fruit weight (g) & 1 & 1 & \begin{tabular}{|l|l}
1 & \\
\end{tabular} & 1 & \begin{tabular}{l|l}
1 & 1 \\
\end{tabular} & \begin{tabular}{l|l}
1 & 1 \\
\end{tabular} & \begin{tabular}{|l|l|}
1 & 1 \\
\end{tabular} & \begin{tabular}{l|l}
1 & 1 \\
\end{tabular} & 1 & 1 & 1 & 1 & 1 & 1 & 1 & 1 & 1 & 1 & 1 & 1 & 1 & 1 & 1 & 1 & 1 & 1 & 1 & 1 & 1 & 1 & 1 & 1 \\
\hline No. of fruit per plant & 5 & 3 & \begin{tabular}{|l|l}
1 & \\
\end{tabular} & 1 & \begin{tabular}{l|l}
3 & 3 \\
\end{tabular} & \begin{tabular}{|l|l|}
3 & 3 \\
\end{tabular} & \begin{tabular}{|l|l|}
5 & 5 \\
\end{tabular} & \begin{tabular}{|l|l|}
5 & 3 \\
\end{tabular} & 5 & 1 & 5 & 3 & 5 & 3 & 1 & 3 & 3 & 3 & 3 & 5 & 5 & 5 & 3 & 3 & 3 & 3 & 5 & 3 & 1 & 3 & 3 & 5 \\
\hline Fruit yield per plant $(\mathrm{g})$ & 7 & 7 & \begin{tabular}{|l|l}
7 & 1
\end{tabular} & 7 & \begin{tabular}{l|l}
7 & 7
\end{tabular} & \begin{tabular}{l|l}
7 & 7
\end{tabular} & \begin{tabular}{|l|l|}
7 & 7 \\
\end{tabular} & \begin{tabular}{l|l}
7 & 7
\end{tabular} & 7 & 7 & 7 & 7 & 7 & 7 & 5 & 7 & 7 & 7 & 7 & 7 & 7 & 7 & 7 & 7 & 7 & 7 & 7 & 7 & 7 & 7 & 7 & 7 \\
\hline No. of seed per fruit & 3 & 1 & 3 & 3 & \begin{tabular}{l|l}
3 & 3 \\
\end{tabular} & \begin{tabular}{l|l}
3 & 3 \\
\end{tabular} & \begin{tabular}{|l|l|}
3 & 3 \\
\end{tabular} & 33 & 3 & 3 & 3 & 3 & 3 & 3 & 1 & 3 & 3 & 3 & 3 & 3 & 3 & 3 & 3 & 3 & 3 & 3 & 1 & 1 & 1 & 3 & 3 & 3 \\
\hline 100 seed weight $(\mathrm{g})$ & 1 & 1 & \begin{tabular}{l|l}
1 &
\end{tabular} & 1 & \begin{tabular}{l|lll}
1 & 1
\end{tabular} & \begin{tabular}{l|l|}
1 & 1 \\
\end{tabular} & \begin{tabular}{|l|l|} 
& 1 \\
\end{tabular} & \begin{tabular}{|l|l|}
1 & 1 \\
\end{tabular} & 1 & 1 & 1 & 1 & 1 & 1 & 1 & 1 & 1 & 1 & 1 & 1 & 1 & 1 & 1 & 1 & 1 & 1 & 1 & 1 & 1 & 1 & 1 & 1 \\
\hline
\end{tabular}


Generally growers prefer round shaped fruit with green colour, sometime oval fruits, so there is ample scope to be bread to fulfill consumer choice using the existing fruit variability of kankoda in Chhattisgarh.

Fruit length was quit variable from (Short, Medium, Long and Very long) the short genotypes fruits coding in KRISHNAPUR, RMF-1, RMF-27, RMF-7-P-1, PHULE-MD5-2, AMBIKA-13-5, RAIGARH, RMF-G-39, AJSG-3 AJSG-4, AJSG-5, INDIRA KANKODA-1, PK-5, PK-26, PK-35, PK-46, PK-49, RMF-17, RMF P-4, PHULE MD 5-1, NDM-1, NDM-5, RMDSG-1, PK-33, AMBIKA-K 12-1, AMBIKA-13-6, NDM-2, NDM-3, NDM-4, DHARAMJAYGARH, and RMF-G-49. Medium long fruits genotypes PK-34 and PK-9.

Conical spine density study revealed that fruits genotypes were found to be conical spine density (Thick and thin) while remaining thick spine density genotypes KRISHNAPUR, RMF-1, RMF-7-P-1, PHULE-MD-5-2, AMBIKA-13-5, RAIGARH, RMF-G-39, INDIRA KANKODA-1, PK-5, PK-26, PK-35, PHULE MD 5-1, NDM-1, NDM-5, AMBIKA-K 12-1, AMBIKA-13-6, NDM-2, NDM-4, DHARAMJAYGARH, RMF-G-49 and PK-9. Thin conical spine density genotypes coding in PK-34, PK-46, PK-49, RMF-17, RMF-27, RMF P-4, RMDSG-1, PK-33, NDM-3, AJSG-3 AJSG-4 and AJSG-5.

Were observed conical spine strength (soft and hard) in conical spine strength soft genotypes PK-34, PK-46, PK-49, RMF-17, RMF-27, RMF P-4, RMDSG-1, PK-33, NDM-3, AJSG-3 AJSG-4 and AJSG-5. Conical spine strength hard genotypes KRISHNAPUR, RMF-1, RMF-7-P-1, PHULE-MD-5-2, AMBIKA-13-5, RAIGARH, RMF-G-39, INDIRA KANKODA-1, PK-5, PK-26, PK-35, PHULE
MD 5-1, NDM-1, NDM-5, AMBIKA-K 12-1, AMBIKA-13-6, NDM-2, NDM-4, DHARAMJAYGARH, RMF-G-49 and PK-9.

The single fruit weight (g) varied from coding (Light, Medium, Heavy and Very heavy) were present in all genotypes as light weight $(<50 \mathrm{~g})$, medium $(51-100 \mathrm{~g})$, heavy $(101-150 \mathrm{~g})$ and very heavy $(>150 \mathrm{~g})$. No of fruits plant ${ }^{-1}$ was classified, viz., (Few <20, Moderate 2140, Many 41-60 and Profuse >60) genotypes PK-49 and KRISHNAPUR yielded fruits and fruits plant ${ }^{-1}$ respectively.

Fruit yield varied from $702 \mathrm{~g}$ (RMF-G-49) to 2691g (KRISHNAPUR). Number of seed fruit $^{-1}$ classified in (Few, Less, Medium, and Many) the genotypes produce 1-15 seeds fruits $^{-1}$ genotypes PK-5, PHULE MD-5-2, DHARAMJAYGARH, RMF-G-39 and RMFG-49 and 16 to 30 seed produced genotype PK-34，PK-46，PK-49，RMF-17，RMF-27, RMF P-4, RMDSG-1, PK-33, NDM-3, AJSG-3 AJSG-4, AJSG-5, KRISHNAPUR, RMF-1, RMF-7-P-1, AMBIKA-13-5, RAIGARH, INDIRA KANKODA-1, PK-26, PK-35, PHULE MD 5-1, NDM-1, NDM-5, AMBIKA-K 12-1, AMBIKA-13-6, NDM-2, NDM-4, and PK-9. Consumers do not prefer the presence of large no of seeds in fruit because it decreases the fruit palatability. So breeder should developed cultivar having fruits with less number of seeds.

Cluster I consisted of genotype namely Indira Kankoda-1, PK -5, PK -9, PK-26, PK-34, PK-35， PK-46， KRISHNAPUR， PK-49, RMF-1, RMF-17, RMF-27, RMF-P-4, RMF7-P-1, PHULLE MD-5-1, PHULLE MD-5-2, NDM-1, NDM-5, RMDSG-1, PK -33, AMBIKA-K-12-1, AMBIKA 13-5, AMBIKA 13-6, RAIGARH, NDM-2, NDM-3, NDM-4, DHARMJAYGARH, RMF-G-39, RMF-G-49 and AJSG-5 having 55 percent similarities whereas, cluster II consisted of AJSG-3 and AJSG-4 having 58 percent similarity with 
cluster I. Cluster II again partitioned into two sub cluster in which first sub cluster of cluster II PK -5, PK-26, PK-34, RMF-17, RMF-27, RMF-P-4, RMF-7-P-1, PHULLE MD-5-1, PHULLE MD-5-2, NDM-1, NDM-5, RMDSG-1, PK -33, AMBIKA-K12-1, AMBIKA 13-5, AMBIKA 13-6, RAIGARH, NDM-2, NDM-3, NDM-4, DHARMJAYGARH, RMF-G-39, RMF-G-49 and AJSG-3. Cluster second sub cluster INDIRA KANKODA-1, PK -9, PK-35, PK 46, KRISHNAPUR, PK-49, RMF-1 having $63 \%$ similarities with cluster II.

Among 29 morphological characters studied, the variation exhibited in day to first flowering, stem and leaf characteristics, fruit and seed size and finally seemed to play major role in separating the genotypes. Sultana et al., (2001) also reported such separation between wild and cultivated species using morphological variation in lablab bean. Based on morphological variation, Hubbard and Garbary (2002) also classified seaweed (Codium spp.) in different subspecies using cluster analysis and principle component analysis. The dissimilarity order present study as observed in dissimilarities matrix. The present study similarities found that Bhadra and Akhtar (1991) in black gram, Natarajan et al., (1998) in green gram, Rasul and Okubo (2004) in kakrol found no relationship with geographical isolation and genetic diversity of the crop.

The basic objective of spine guard characterization is to test the occurrence of traits that help in identifying a particular genotypes. Morphological traits are not influenced by the environment; transfer of those characters is fully governed by genes and could be plant breeder use in hybridization program. From this point of view, morphological characterization of leaf, fruit, flower and seed characters were performed (Rahman, 2015). The genetic variation suggested that a positive response to direct selection is possible for all the traits studies (Tiwari and Tigga, 2015). Morphological characterization indicated the selection for number of stem per plant, number of fruit per plant, single fruit weight could be selection criteria for simultaneously increasing fruit yield. However morphological description alone may be not sufficient for characterization, their some other description based on biochemical and molecular marker called be considered for robust result and development of selection criteria.

\section{References}

Basumatary, P., Bora, G.C., Kalita, U.C., Saikia, L. and Deka, N. C. (2014) Variability and correlation studies in spine guard (Momordica dioica Roxb.). Journal of Agriculture and food science (2), 77-81

Bhadra, S.K and M.I. Akhtar (1991) Genetics divergence for yield and its morphological components of mungbean (Vigna radiate (L) wilczek). SABRAO journals, 23: 127-136.

Brown, R.N. and Myers, J.R. (2002) A genetic map of squash (Cucurbita sp.) with randomly amplified polymorphic markers and morphological Markers. $J$. Amer. Soc. Hort. Sci., 127:568-575.

Grant, M. L., Miller D. M. and Culham A. (2001) Genetic and morphological analysis of the origin and identity of perennial lavatera (Malvaceae) cultivars. J. Amer.soc. Hort. Sci., 125:593-598.

Kushwaha, S.K., Jain, A., Gupta, V.B., and Patel, J.R. (2005) Hepatoprotective activity of the fruits of Momordica dioica, Nigerian journals of natural product and Medicine, (9), 29- 31

Masud, M. A. T., Chowdhury, M. A. Z., Hossain, M. A., and Hossain, S. M. M. (1995) Multivariate analysis in pumpkin 
(cucurbita moschata Duch ex poir) Bangladesh J. Pl. breed. Genet. 8:45-50 Mathew L, RC Babu, J., Souframanien, P Chezhian, P., Shanmugasundaram, P., Nagarajan and S., Sadasivam (2000) DNA polymorphism among rice (Oryza sativa L.). accessions differing in drought tolerance. J. Plant Biol. 27: 145-152.

Natarajan, C.K., Tiagarajan and R. Rathanaswamy (1998) Association and genetics diversity studies in black gram. Madras agric. J., 75: 238-245.

Rahman, M. M., Rasul, M. G., Islam, M. S., Sultana N., and Hossain, M.A. (2001) Genetic diversity in capsicum $s p$. Bangladesh J. Pl. Breed. Genet, 14:7-11

Rahman, M., (2015) Morphological characterization and diversity analysis in bitter gourd (Momordica charantia 1.) M.Sc. thesis Genetics and Plant Breeding sher-e Bangla univ. Dhaka, 93-95.

Raj, N. M., Prasanna, K. P., Peter, K. V. (1993) Momordica spp. In kallo G. Berge Bo (Eds). Genetic improvement of vegetables crops. Pergamon press; Oxford, 239-243.

Rakh MS, Chaudhari SR (2010) Literature survey of plant Momordica dioica
Roxb. Willd. An update. Int. J. Pharmacol Res. Develop. 1-8

Rashid, M. M. (1976) Vegetable in Bangladesh (In Bengali) $1^{\text {st }}$ Edn. Bangla Academy, Dhaka Bangladesh. 494.

Rasul, G.M. and Okubo, H. (2004) Morphological and Physiological Variation in Kakrol (Momordica dioica Roxb.) J. Fac. Agr., Kyushu univ., 49 (1), 1-11.

Rasul, M. G., Hiramatsu, M., and Okubo, H. (2003) Morphological and physiological variation in kakrol (Momordica dioica Roxb.), J. Fac. Agr Kyushu Univ, 49(1), 1-11.

Rohlf FJ (1998) NTSYS-pc: Numerical taxonomy and multivariate analysis system, version 2.02. Exter Software.

Sultana, N., Ozaki Y., and okubo, H. (2001) Morphological and physiological variation in lablab bean (Lablab purpureus (L) Sweet.). J. Fac. Agr., Kyushu Univ., 45: 465-472.

Tiwari, J.K. and Tigga, K. (2015) Genetic architecture and correlation analysis for fruit yield in different genotypes of spine gourd (Momordica dioica Roxb.) Progressive Research - international journal, 10 (4), 2425-2428

\section{How to cite this article:}

Sanjay Bhagat, S.K. Sinha and Tiwari, J.K. 2017. Identification and Evaluation of MorphoPhysiological Variation in Spine Gourd (Momordica dioica Roxb.). Int.J.Curr.Microbiol.App.Sci. 6(11): 531-540. doi: https://doi.org/10.20546/ijcmas.2017.611.064 